\title{
Driver Rating: a mobile application to evaluate driver behavior
}

DOI: $10.46932 / \mathrm{sfjdv2n2-001}$

Received in: November 1st, 2020

Accepted in: December 30th, 2020

\section{Nielson S. Trindade}

Master - Salvador University - UNIFACS.

Full Address: UNIFACS - R. Dr. José Peroba, 251 - Stiep, Salvador - BA, 41770-235

E-mail: nicoctrindade@gmail.com

\section{Artur H. Kronbauer}

Doctor. Salvador University - UNIFACS e Bahia State University - UNEB.

Full Address: UNIFACS - R. Dr. José Peroba, 251 - Stiep, Salvador - BA, 41770-235

E-mail: arturhk@unifacs.br

\section{Helder G. Aragão}

Master - Salvador University - UNIFACS e Estacio-Bahia University Center Estácio - Estácio FIB.

Full Address: UNIFACS - R. Dr. José Peroba, 251 - Stiep, Salvador - BA, 41770-235

E-mail: helderaragao@gmail.com

\section{Jorge Campos}

Phd - Salvador University - UNIFACS e Bahia State University - UNEB.

Full Address: UNIFACS - R. Dr. José Peroba, 251 - Stiep, Salvador - BA, 41770-235

E-mail: jorge@unifacs.br

\begin{abstract}
The combination of data from sensors embedded in vehicles and smartphones promises to generate great innovations in intelligent transportation systems. This article presents Driver Rating, a mobile application to evaluate the behavior of drivers based on the data gathered from vehicles' and smartphones' sensors. The Driver Rating application analyzes five variables (fuel consumption, carbon dioxide emission, speed, longitudinal acceleration, and transverse acceleration) to evaluate driver's behaviors while driving. To test the Driver Rating application and identify its potentialities, an experiment was carried out on an urban environment, showing promising results regarding the classification of drivers' behavior.
\end{abstract}

\section{INTRODUCTION}

Responsible driving of auto-vehicles has a direct impact not only on pedestrian, driver, and passenger's safety, but also affects the economy, the environment, and public health. The driver's misbehavior accounts for more than half of all road accidents in the United States (AAA Foundation for Traffic Safety, 2009). Constant lane changes, speeding, sudden acceleration and inadvertently braking are some of the drivers' behaviors that affect traffic safety and fuel consumption. Aggressive driving style increases energy consumption by up to 20\% (Araújo et al., 2012) (Meseguer et al., 2013), which implies an increase of emission of greenhouse gases at the same proportion. Greenhouse gases and pollutants have a huge impact on the environment and the health of citizens (Barth et al., 2008). Thus, the production chain of fossil fuels has the potential to affect not only the urban environment but the climate on a global scale 
(Meseguer et al., 2013).

Regarding environmental impacts, the use of electric or hybrid vehicles promises to solve the problem in the long term. Regarding the safety of transport system users and citizens, however, the solution to the problem will only be effective with the use of completely autonomous vehicles. In the short term, conducting public education campaigns for drivers, increasing enforcement and imposing high traffic fines have been the measures used to try to create collective awareness and mitigate the negative impact of driving.

Aiming at helping drivers to be aware of their drivers' styles and behaviors, this paper presents Driver Rating, a mobile application to evaluate the behavior of drivers based on data gathered from sensors embedded in motor vehicles and smartphones. The Driver Rating application analyzes the behavior of the driver based on five variables: speed, fuel consumption, braking or sudden accelerations, aggressive curves, and the level of emission of greenhouse gases.

Data about the fuel consumption, speed, and emission of greenhouse gases are gathered from the vehicle's ECU (Engine Central Unit) via an OBD (On-Board Diagnostics) interface. Longitudinal and transverse accelerations are obtained from sensors embedded in smartphones. Finally, the current position of the vehicle is gathered from the smartphone location sensor and the maximum speed allowed on the road is obtained from data stored locally on the smartphone or from some map Web services. The Driver Rating application takes all these pieces of information and rates the driver at regular time's interval. To validate the Driver Rating application, some tests were carried out on a real case scenario.

The remainder of this paper is structured as follows: Section 2 discusses some works that use sensors embedded in vehicles and smartphones to evaluate the drivers' behavioral patterns. Section 3 describes the architecture and implementation of the Driver Rating mobile application and discusses the variables used in the classification of drivers. Section 4 presents the results of a field experiment of the Drive Rating application. Section 5 concludes and indicates future works.

\section{RELATED WORK}

The association of data from sensors embedded in vehicles with smartphone sensors, combined with the processing power, storage capacity and ease of Internet connection of these devices, promise to generate great technological innovations in the area of Intelligent Transportation Systems (ITS) and to improve the driver style (Alvear et al., 2015).

One of the first works to explore the synergetic union of vehicular and smartphones data was proposed by (Zaldivar et al., 2011). This paper proposes something like a smart black box. The application stores data from the accelerations suffered by the vehicle when the trigger of the airbags runs off. Once an accident is identified, the application sends a warning through the email service or short message service with information about the event, such as time, location and an estimate of the severity of the accident based on the degree of deceleration experimented by the vehicle. 
The work of (Amarasinghe et al., 2015) proposes a mobile application to capture several vehicular variables to detect anomalies in the vehicle operation and the prediction of failures. Vehicular variables are collected through readings of the ECU via OBD interface. Besides the misfunctioning of the vehicle, the application is also able to identify, in real-time, imprudent driving modes, issuing warnings and driving alerts. The imprudent driving behavior is identified employing vehicle variables only, that is, it does not exploit the profusion and richness of smartphones' sensors.

Other studies seek to identify an aggressive behavioral pattern of the driver based on the accelerations experienced by the vehicle. Aggressive driving can be defined as the act of braking or starting the movement of the vehicle in an abrupt way, the act of performing turns with high speed and changing lanes suddenly and repetitively. These behaviors, coupled with other equally imprudent acts, are responsible for most road accidents. An example of this approach is the work of (Bhoyar et al., 2013) that identifies steering patterns based on the comparison of the accelerations obtained from the accelerometers of a smartphone with the signatures of accelerations of various behavioral patterns stored in its database. Once an acceleration pattern compatible with aggressive steering has been identified, the application issues an alert to the driver.

Despite the technological improvements experienced by motor-vehicles in recent decades and the development of fewer pollutant fuels, road transport continues to be the major responsible for air pollution in urban areas. Thus, it is always important to develop applications that associate vehicle consumption with the behavioral pattern of the driver. The idea is if a fossil fuel-based vehicle is bad, this same vehicle when misoperated is even worse. (Meseguer et al., 2013) proposes a methodology to calculate, in real-time, the consumption and environmental impact of gasoline and diesel engines. The methodology uses data from the electronic vehicle control unit (ECU). The vehicle variables used in the process involve speed, fuel flow rate, air mass rate, internal engine pressure, among others. The methodology proposed by (Meseguer et al., 2013) uses neural networks to classify driver behavior in the following ranges: normal, quiet and aggressive. The work also demonstrated that the classification of driver behavior directly affects fuel consumption.

Another relevant work aiming at reducing fuel consumption that combines vehicular and smartphones data is the Driving Coach application (Araújo et al., 2012). Driving Coach was developed in a three-layer architecture. The bottom layer is responsible for collecting vehicle and smartphone data (e.g., speed, fuel consumption, acceleration). The middle layer uses Fuzzy Logic to convert the values of the variables obtained in the lower layer to classify fuel consumption and driver behavior. Finally, the last layer provides the driver with the results of the evaluation and suggests, in real-time, the action to be taken to reduce fuel consumption.

The proposal of this work is the development of a mobile application to evaluate the drivers' behavioral patterns based on sensors embedded in vehicles and smartphones. Our approach is similar to the technique used in Driving Coach (Araújo et al., 2012) and DrivingStyles (Meseguer et al., 2013). An important difference in our work is that we use five dimensions in the characterization of the behavioral 
pattern: longitudinal and transverse acceleration, fuel consumption, velocity and emission of greenhouse gases. To the best of our knowledge, no initiative uses all these pieces of information to identify and classify the behavioral pattern of the drivers. Another important difference from previous works is that we classify the driving style in a broader way, that is, our application rewards with a good grade not only the motorist that drives safely but also the driver that have decided to buy an economy car instead of a fuel-inefficient car and uses less pollutant and environmental-friendly fuels. The next section discusses all the variables used for classifying drivers.

\section{ARCHITECTURE AND IMPLEMENTATION OF DRIVER RATING}

The Driver Rating application has a three-tier architecture. The lowest layer is responsible for communicating with in-vehicle and smartphone's embedded sensors and data persistence. The intermediate layer corresponds to the Fuzzy classifier of the driver's behavior. The third layer contains the graphical user interface.

The Driver Rating activity flow starts when the driver initiates the process of data collection and evaluation. Figure 1 illustrates the analytical scheme of the logical flow stages of the Driver Rating application. Driver Rating uses the GPS receiver as a time ticker to collect data from all sorts of embedded sensors and Web services (Figure 1.a). Whenever a new position is provided by the application location service, the application requests data from the OBDUpdate, OverpassUpdate, and AccUpdate modules (Figure 1.b, 1.c, and 1.d). All data coming from theses modules are georeferenced and stored in main memory for a certain amount of time before they are sent to a persistent database. The amount of time can be defined in the application configuration module. By default, the time window is set to 300 seconds, meaning that the driver's behavior will be evaluated at 5 minutes intervals. 
Figure 1: Analytical diagram of the logic flow stages of the Driver Rating application.

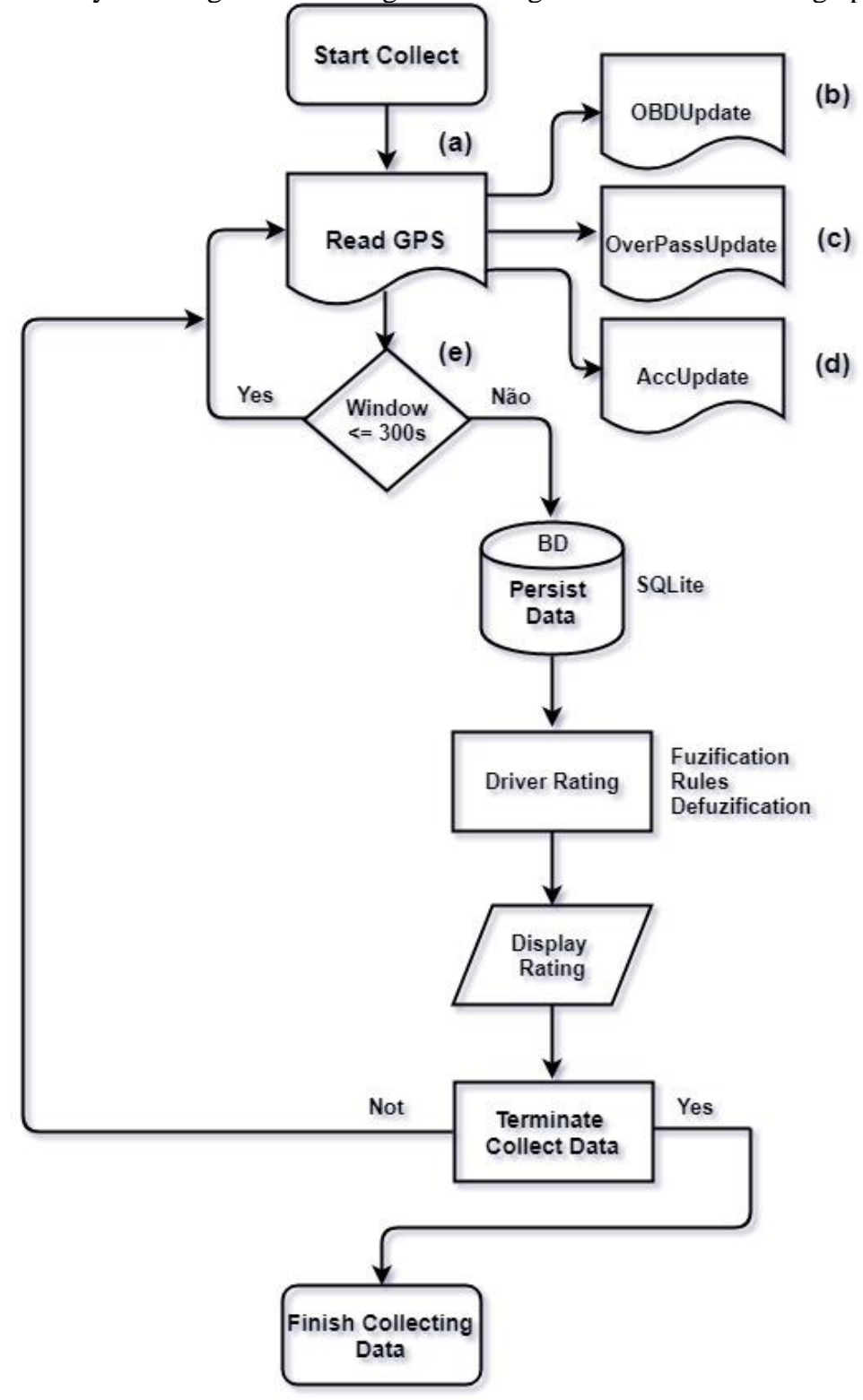

Driver rating uses five dimensions to evaluate the driver's behavioral patterns (i.e., longitudinal and transverse acceleration, fuel consumption, velocity, and emission of greenhouse gases). All pieces of information needed to perform the evaluation come from OBDUpdate, OverpassUpdate, and AccUpdate modules.

The OBDUpadate module (Figure 1.b) is in charge to collect data to evaluate the fuel consumption and emission of pollutant gases. Before start reading the data, the OBDUpadate module establishes a Bluetooth connection between the application and the OBD reader and tests the communication between the OBD reader and the vehicle's ECU.

The average fuel consumption is computed between every two location readings coming from the location service. This measure, however, is not obtained directly from the ECU. To compute fuel consumption, the application needs to read from the ECU the number of Revolution per Minute (RPM), the 
Input Air Temperature (IAT), the Intake Manifold Absolute Pressure (MAP), and the Fuel Flow measured in liters per hour. The result of this computation is used to rate the driving behavior regarding fuel consumption and emission of greenhouse gases.

Regarding the evaluation of the driver based on vehicle fuel consumption, the value of this variable is standardized to consider the type of vehicle being used. Driver Rating configuration process requires that drivers select the model of the vehicle they are driving from a list. The list of vehicles is populated with information extracted from the Inmetro database (Inmetro, 2018). Inmetro database has all vehicles' models sold in Brazil, the expected fuel consumption, an estimated amount of pollutant gases emitted by each model, among other things. Thus, the standard fuel consumption is obtained by dividing current fuel consumption by the expected fuel consumption, as indicated by Inmetro. Values less than 1 indicate that the vehicle is being driven efficiently from an energetic point of view, while values greater than 1 indicate excessive fuel consumption. Drive Rating use the standard fuel consumption to downgrade those drivers who spend more fuel than expected for the model of the vehicle it drives.

Another important information defined in the configuration process is the kind of fuel being used (diesel, alcohol, gasoline or any combination of the latter two). Driver rating uses the type of fuel, the actual fuel consumption, and the volume of pollutants emitted by each vehicle model to compute the total amount of pollutants released in the atmosphere. Driver Rating uses this value to evaluate the driver concerning the variable emission of gases. Thus, the higher the fuel consumption of the vehicle, the worse the driver's rating for this variable. This grade is further adjusted to incorporate two penalties that do not properly concern the driver's way of driving, but his attitude towards the environment. The choice of a vehicle that pollutes the environment less, such as hybrid or electric vehicles, and the option to use non-fossil fuels are both considered and alleviate the penalty applied to drivers.

The first penalty applied to the emission of gas variable reflects the choice of the type of vehicle made by the driver. For this matter, it is measured how far away is the actual emission of gases from the emission of gases of the reference car. We used as reference the lowest value of $\mathrm{CO} 2 / \mathrm{km}$ found in the table of the Inmetro. If the vehicle used is the reference car, a hybrid vehicle that generates only 71 grams of $\mathrm{CO} 2 / \mathrm{km}$, there is no penalty. If the driver owns a sports car that produces an impressive 274 grams of $\mathrm{CO} 2 / \mathrm{km}$, the penalty downgrades the value of this variable by a factor of 3,85 .

The second penalty is based on the choice of fuel used in the vehicle. Vehicles with "flex" engines can be evaluated differently according to the kind of fuel used. Drivers who only use alcohol, for example, produces fewer pollutants. In this way, these drivers are less penalized than drivers who use gasoline only. Remember that vehicles that use alcohol, gasoline and diesel always suffer a penalty, as their combustion engines still emit pollutants in the atmosphere. Currently, only electric vehicles do not suffer any penalty in this variable.

The OverPassUpdate module is responsible to monitor the speed of the vehicle (Figure 1.c). The 
variable speed is obtained redundantly since it can be read both from OBDUpdate module and from the location service of the smartphone. For classification purposes, the speed is compared with the maximum speed allowed for the track. The maximum speed is obtained through the Overpass project, hosted by Open Street Map (Haklay et al., 2008) (Sperandio et al). The OverPass API allows mobile applications to retrieve relevant information about the local in the road network where the vehicle is passing by Among the information retrieved by the API, the Driver Rating application uses only the current track speed that is compared to the vehicle speed to evaluate driver behavior. The use of the Overpass API requires an Internet connection to evaluate driver behavior in real-time (Dantas et al., 2017).

The AccUpdate module (Figure 1.d) register the highest longitudinal and transverse accelerations achieved by the sensors (accelerometers) embedded in the smartphone that runs the Driver Rating application. Longitudinal and transverse accelerations experienced by the vehicle are the variables most used in applications that aims to classify drivers behavioral pattern. The longitudinal acceleration captures the movement of braking and departing of the vehicle, while the transverse acceleration identifies the execution of turns. Driver Rating considers an Aggressive steering behavior if the vehicle experience accelerations greater than $0.4 \mathrm{G}$, a normal behavior if the acceleration lays in the interval between $0.2 \mathrm{G}$ e $0.4 \mathrm{G}$, and a smooth driving style for accelerations less than $0.2 \mathrm{G}$.

At regular time intervals, all variables collected by the OBDUpdate, OverpassUpdate, and AccUpdate modules are persisted in a local database and submitted to a Fuzzy classifier (Figure 1). The fuzzy classifier translates the values obtained from vehicle sensors and smartphones into a grade, which varies from 1 to 4 , and a concept, represented by the most appropriate language terms for each variable. The language terms Bad, Medium and Good are used for the variables fuel consumption and speed. Variables related to longitudinal and transverse accelerations are classified as Caution, Moderate and Risky. Finally, gas emission uses the terms Red, Yellow, and Green.

The presentation layer allows drivers to visualize a summary with grade obtained for every variable individually (Figure 2.a) or the history of the evaluation of each variable depicted in a map (Figure 2.b). Inspecting the map each variable, for instance, it is possible to identify road segments where the driver was poorly evaluated for driving above the speed limit or which curve was performed aggressively. The next section shows the results of a field experiment with the Driver Rating application. 
Figure 2: Screenshots of the driver rating application. a) summary table with grades and classification terms obtained for each control variable and $b$ ) history of the evaluation of a control variable depicted in a map.

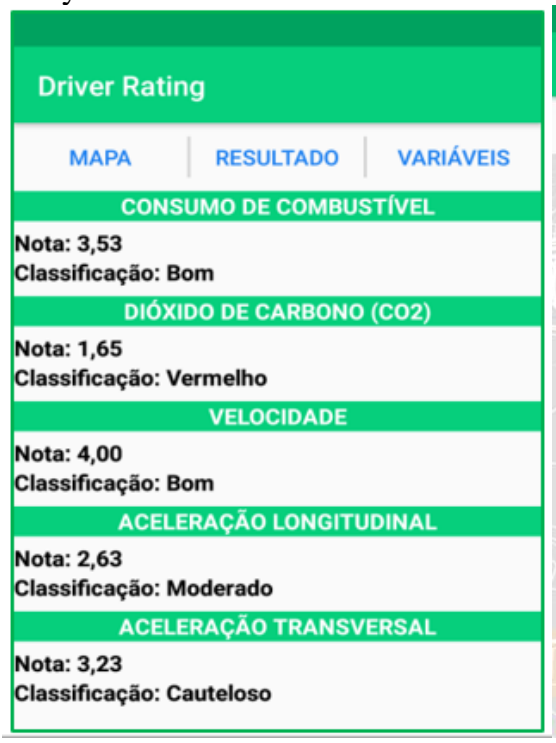

a)

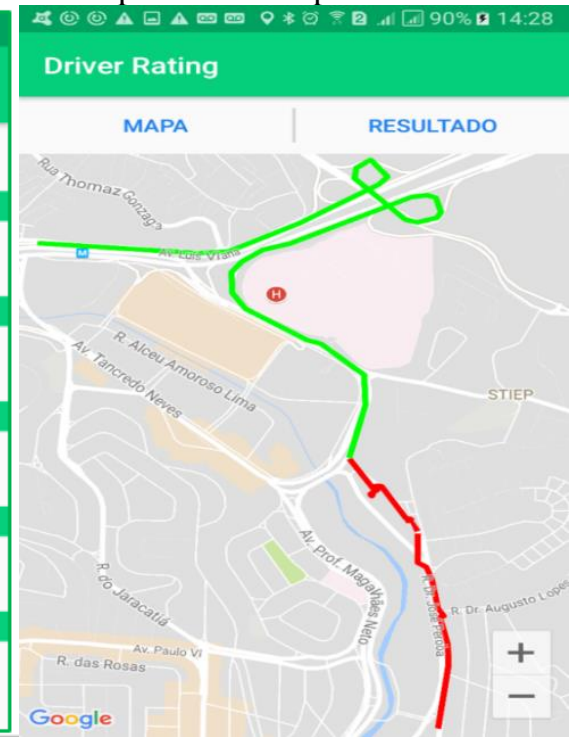

b)

\section{FIELD EXPERIMENT}

Aiming at testing the use of Driver Rating in real case scenarios, we conduct a field experiment with four different kinds of vehicles, two different models of OBD readers, and two smartphones equipped with the Android operating system. The field experiment was designed to evaluate Driver Rating's classification capacity regarding all variables (fuel consumption, carbon dioxide emission, speed, longitudinal acceleration, and transverse acceleration). To achieve this goal, some situations and drivers' behaviors were induced. Due to the limited space in this paper, we have decided to present only some illustrative tests.

Fuel Consumption is a variable that can be affected by external factors (e.g., traffic jams and steep ramps) and behavioral factors (e.g., aggressive or imprudent behaviors). To analyze the Driver Rating classification process regarding fuel consumption, two experiments were carried out: an experiment passing through a road segment with traffic congestion and another experiment going up and down a long steep ramp. The driver performed both experiments with a smooth driving style (i.e., without forcing the vehicle engine, respecting speed limits, and without sudden steering changes or abrupted braking.

Maps in Figure 3 show the route made by the vehicle during the experiments. These maps are part of the Driver Rating application and are used to present historical data concerning drivers' behaviors. The trajectory of the vehicle is colored accordingly the grade obtained for a specific variable. Regarding fuel consumption, for instance, the green traces indicate low fuel consumption, yellow traces illustrated fuel consumption near the fuel consumption suggested by the manufacturer, and red indicates excessive fuel consumption. Driver Rating allows the evaluation of any variable of any period, individually or combined. In this way, drivers can analyze their behavioral profiles both in time and space.

The first experiment started in a traffic jam (Figure 3.a). The Driver Rating application identified 
high fuel consumption at this stretch of vehicle trajectory and assigned a negative grade for this variable (red trace). The evaluation of the fuel consumption was positive in segments of the road where there was no traffic congestion (green trace).

Driver Rating application while evaluating the fuel consumption of the vehicle going up and down steep ramps. The map on Figure 3.b depicts the trajectory of the vehicle during the experiment. A black dashed line was drawn to indicate the stretch of the road with a long steep ramp. In this experiment, the vehicle passes through the ramp twice, that is because there are two traces along the ramp (one red and another yellow/green). The vehicle begun its trajectory somewhere on the bottom of the map, went up the ramp, traveled on a plain segment of the road, made a U-turn (top of the map), travel again through the plain segment, and went down the ramp.

When the vehicle was going up the ramp, the fuel consumption was very high. A red trace depicts this situation. When the vehicle traveled along the plain segment without traffic congestion the trace of the vehicle has a green color, indicating that the fuel consumption is the same or better than the estimated fuel consumption for this vehicle. When the vehicle was going down the ramp, the trace of the vehicle was initially green but became yellow on the second half of the ramp. The mixed evaluation of fuel consumption of the vehicle while going down the ramp could be motivated by the fact of the engine-brake was used during all way down, thus increasing fuel consumption.

Figure 3: Presentation of the results of an experiment to evalauate fuel consuption. a) driving in congested road a segment and b) going up and down on a long steep ramp.

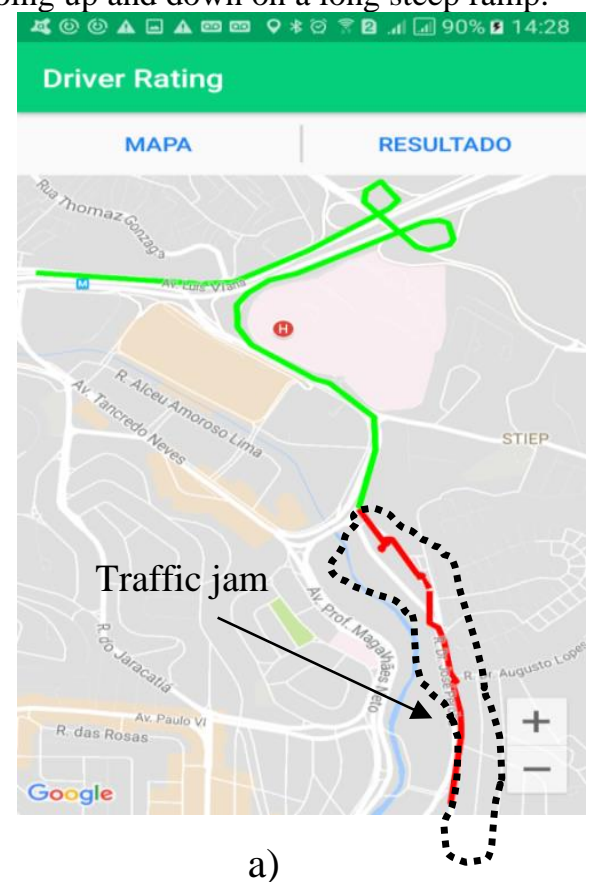

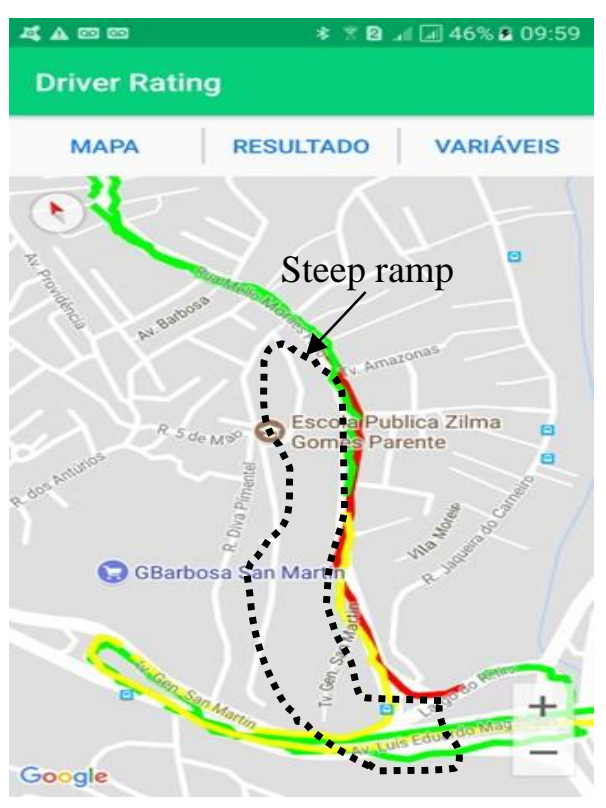

b)

The first two experiments evaluated the driver behavior based on external factors (i.e., traffic jams and ramps). We recognize that the evaluation of the behavioral profile of the driver considering these factors 
may be unfair. External factors, since when they can't be avoided, could be considered unrelated to the driver's way of driving. We are looking at ways of considering external causes but diminishing the weight of them on the evaluation system.

Aiming at verifying the impact of internal factors in the driver behavior evaluation, we conduct two other experiments: the first experiment tested the sensitivity of the application to evaluate the aggressive behaviors on curves. The second experiment evaluated speeding. The trajectory of the vehicle in the first experiment started with the green trace on the bottom of the map (Figure 4.a). The second segment of the vehicle trajectory (red trace) represents the fact that the driver performed a sharp left curve with high speed. The third segment of the vehicle trajectory (yellow trace) represents the fact that the driver performed another sharp left curve but with moderate speed. By executing the curve aggressively, the driver may have overpassed the speed limit. The map on figure 4.a, however, depicts the result of the variable transversal acceleration alone.

Figure 4: Presentation of the results of experiments to evalauate internal factors. a) driving aggressively on curves and b) driving over the speed limit.

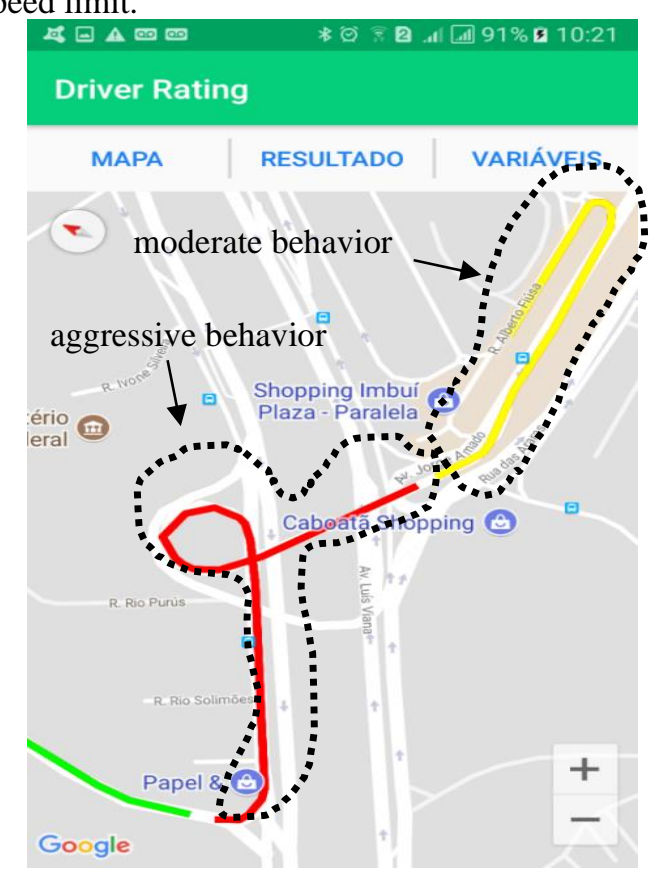

a)

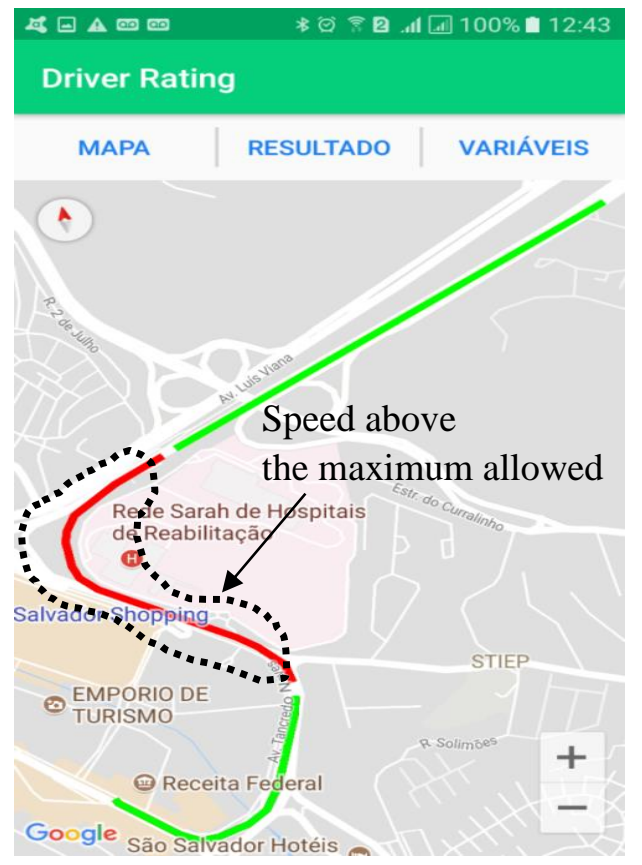

b)

It is worth noting that the behavioral evaluation is not punctual but valid throughout periods of observation. The observation time window can be configured by the user in the settings screen of the Driver Rating application. A time window of 2 minutes, for example, implies that the application will collect information from the sensors during this period, but will only rate the driver's behavior at the end of the time window. In this way, the acceleration peak was probably measured in the middle of the curves, but Driver Rating extends the behavioral evaluation for the entire segment encompassed by the length of the time 
window. Users can increase the frequency of evaluation by setting the time window to lower intervals (up to 1 second), but a shorter time window will increase the processing load on your smartphone and will compromise persistent memory and battery life.

The second experiment tested the Drive Rating while evaluating the speed of the vehicle. For this variable, the Driver Rating application requires an Internet connection. Connectivity is required to obtain the maximum permissible speed on the vehicle location. Every time the location service of the application obtains a new location, a Web request is made through the OverPass API. Another option would be to install a copy of the region's Open Street Map database. This strategy dispenses the Internet connection but will overload the mobile device's persistent memory.

During the second experiment, the driver initially conducts the vehicle at a speed below the speed limit of the road segment. This fact is represented by the green trace on the bottom of the map (Figure 4.a). Suddenly, the driver accelerates the vehicle and reach a speed above the speed limit of the road (red trace on the map). After a while, the driver slows down to a speed below the speed limit and the speeding evaluation becomes green again.

The third group of experiments deals with the evaluation of drivers' behaviors and consumers' choices. The evaluation concerning emissions of pollutant gases uses two kinds of vehicles. The first vehicle's CO2 emission is $145 \mathrm{~g} / \mathrm{km}$ (Figure 5.a) and the second vehicle's CO2 emission is $95 \mathrm{~g} / \mathrm{km}$ (Figure 5.b).

Figure 5: Presentation of the results of experiments to evaluate gas emissions. a) evaluation of a vehicle with a CO2 Emission of $145 \mathrm{~g} / \mathrm{km}$ and b) evaluation of a vehicle with a CO2 Emission of $95 \mathrm{~g} / \mathrm{km}$.

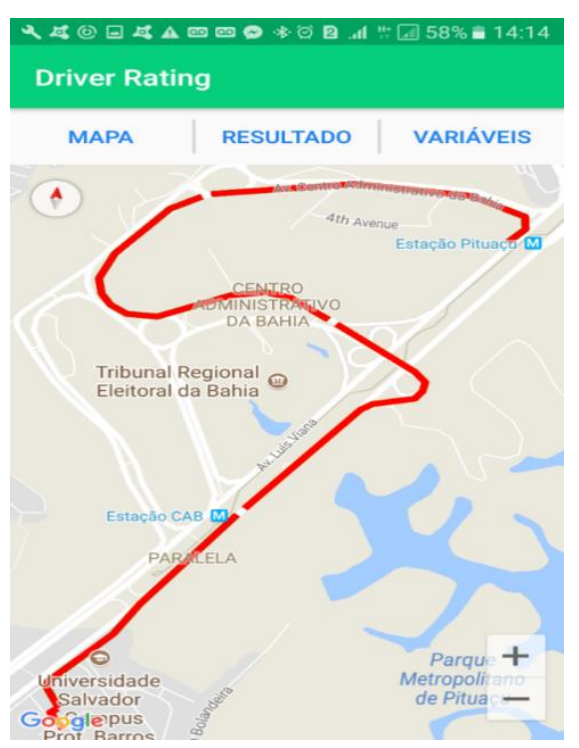

a)

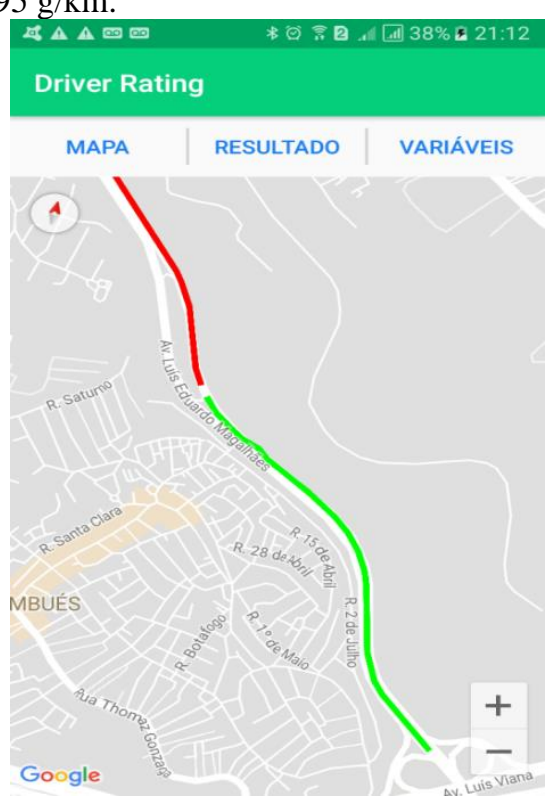

b)

The evaluation of the variable emission of pollutant gases is computed considering the $\mathrm{CO} 2$ emission 
of a reference car. The reference car is the less pollutant vehicle found in the Inmetro database. The map in Figure 5.a depicts the evaluation of a vehicle that emits 145 grams of $\mathrm{CO} 2$ per kilometer. This model emits more than twice the amount of $\mathrm{CO} 2$ than the reference vehicle, which is a hybrid car. Thus, the driver's behavior will always be considered inadequate (red trace), reflecting the non-environmentally-friendly choice of the driver to buy a vehicle that is known to be a polluter.

The map in Figure 5.b shows the evaluation of a vehicle that emits 95 grams of $\mathrm{CO} 2$ per kilometer. This vehicle emits only 50\% more CO2 than the reference vehicle. The evaluation of vehicles with low $\mathrm{CO} 2$ emissions resembles the evaluation of fuel consumption variable, downgraded by a penalty factor proportional to the amount of $\mathrm{CO} 2$ that surpasses the amount of $\mathrm{CO} 2$ emitted by the reference car. Above a certain threshold, it is impossible to get a good evaluation in this variable.

The experiments carried out are proofs of concept and aim to demonstrate the operation of the application in some induced scenarios. We would like to record that all the experiments were carried out by the researchers involved in the project. At no time volunteer drivers conducted the vehicles.

\section{CONCLUSIONS AND FUTURE WORK}

This paper presented Driver Rating, a mobile application for classifying drivers according to data obtained from vehicle sensors and smartphones. The Driver Rating application analyzes and evaluates five variables: fuel consumption, carbon dioxide emission, speed, longitudinal acceleration, and transverse acceleration.

The Driver Rating application aims at encouraging people to drive safely. The Driver Rating application is the digital version of the well-known sign stamped in many vehicles with the phrase "How am I driving?" followed by a contact phone number. In the analog version, the evaluation is done by a person that has witnessed some irregularity practiced by the driver. Thus, the "how am I driving" system is based on moral integrity, goodwill and the ability of the complainant to evaluate the situation. In the digital version, various dimensions of eventual irregularities practiced by the driver are evaluated, exempts eyewitnesses and records the date, time and place of inappropriate behaviors in a continuous and omnipresently way.

The Driver Rating application can be used by any person or company interested in evaluating drivers' behavior. This includes private drivers, taxi drivers, car rental companies, public bus transportation system companies and fleet managers of logistics companies.

As future work, we intend to collect data from a group of users of the Driver Rating application. The objective is to analyze and validate the application as a tool to determine the behavioral pattern of drivers in real situations. We are also considering a way of minimizing external factors in the evaluation of the behavioral pattern of the drivers. Congestion, for example, can be identified by querying the traffic layers of Web map systems, such as Google Maps and Bing Maps. In this way, stretches with congestion would not penalize the driver regarding the excessive consumption of fuel and emission of greenhouse gases 


\section{REFERENCES}

AAA Foundation for Traffic Safety. 2009. “Aggressive Driving: Research Update.” Aggressive Driving: Research

Update. https://www.aaafoundation.org/sites/default/files/AggressiveDrivingResearchUpdate2009.pdf.

Alvear, Oscar, Carlos T. Calafate, Juan Carlos Cano, and Pietro Manzoni. 2015. "Validation of a Vehicle Emulation Platform Supporting OBD-II Communications." 2015 12th Annual IEEE Consumer Communications and Networking Conference, CCNC 2015: 880-85.

Amarasinghe, Malintha et al. 2015. "Cloud-Based Driver Monitoring and Vehicle Diagnostic with OBD2 Telematics." In IEEE International Conference on Electro Information Technology, IEEE, 505-10. http://ieeexplore.ieee.org/lpdocs/epic03/wrapper.htm?arnumber=7293433.

Araújo, Rui, Ângela Igreja, Ricardo De Castro, and Rui Esteves Araújo. 2012. "Driving Coach: A Smartphone Application to Evaluate Driving Efficient Patterns." IEEE Intelligent Vehicles Symposium, Proceedings 1(1): 1005-10.

Barth, M, and K Boriboonsomsin. 2008. "Real-World CO_2 Impacts of Traffic Congestion.” Transportation Research Record: Journal of the Transportation Research Board 2058(1): 163-71.

Bergasa, Luis M. et al. 2014. "DriveSafe: An App for Alerting Inattentive Drivers and Scoring Driving Behaviors." IEEE Intelligent Vehicles Symposium, Proceedings (Iv): 240-45.

Bhoyar, Vaibhav et al. 2013. "Symbian Based Rash Driving Detection System.” 2(2): 124-26.

Dantas, Daniel, Antônio A. de A. Rocha, and Marcos Lage. 2017. "Extracting bus lines services information from GPS registries". 2017. Proceedings of the 23rd Brazillian Symposium on Multimedia and the Web. ACM, 389-397. https://dl.acm.org/citation.cfm?id=3126858.312688.

Haklay Mordechai and Patrick Weber. 2008. “Openstreetmap: User-generated street maps.” IEEE Pervasive Computing, Vol. 7 (4), 12-18. DOI: 10.1109/MPRV.2008.8.

Inmetro. Instituto Nacional de Metrologia, Qualidade de Tecnologia. Disponível em: http://www4.inmetro.gov.br/. Último acesso em: 04/05/2018.

Lakatos, Eva Maria, Marina de Andrade Marconi. "Fundamentos de metodologia científica". 5. ed. São Paulo: Atlas, 2003.

Meseguer, Javier E., Carlos T. Calafate, Juan Carlos Cano, and Pietro Manzoni. 2013. "DrivingStyles: A Smartphone Application to Assess Driver Behavior." Proceedings - International Symposium on Computers and Communications: 535-40.

Solomon, Susan, Gian-Kasper Plattner, Reto Knutti, and Pierre Friedlingstein. 2009. "Irreversible Climate Change due to Carbon Dioxide Emissions." Proceedings of the National Academy of Sciences 106(6): 17049. http://www.pnas.org/lookup/doi/10.1073/pnas.0812721106.

Zaldivar, Jorge, Carlos T. Calafate, Juan Carlos Cano, and Pietro Manzoni. 2011. "Providing Accident Detection in Vehicular Networks through OBD-II Devices and Android-Based Smartphones." Proceedings - Conference on Local Computer Networks, LCN (May 2016): 813-19.

Sperandio V. G.; Dias, V. E. C.; Stempliuc, S. M.; Lisboa-Filho, J. Creating municipal databases from 
OpenStreetMap:

the

conceptual

In: SIMPÓSIO BRASILEIRO DE GEOINFORMÁTICA (GEOINFO), 19, 2018, Campina Grande. Anais... Campina Grande: MCTIC/INPE, 2018. p.25-35. 